\title{
The diets of sows with fish oil might decrease the oxidative stress and inflammatory response in sows, but increase the susceptibility to inflammatory response in their offspring
}

\author{
Wenli Luo \\ Shanghai Jiao Tong University \\ Jing Zhang \\ Shanghai Jiao Tong University \\ Weina Xu \\ Shanghai Jiao Tong University \\ Jianxiong Xu ( $\nabla_{\text {jxxu1962@sjtu.edu.cn ) }}$ \\ Shanghai Jiao Tong University
}

Research

Keywords: Sow, Piglet, Oxidative stress, cytokine, Liver

Posted Date: June 5th, 2020

DOI: https://doi.org/10.21203/rs.3.rs-32903/v1

License: (c) (i) This work is licensed under a Creative Commons Attribution 4.0 International License.

Read Full License 


\section{Abstract}

Backgroup: This aim of this study was to investigate that the effect of supplement maternal diet with fish oil on the oxidative stress and inflammatory response of sows and their offspring.

Methods: Twelve sows were divided into two groups. Sows were fed soybean oil diet (SD) or soybean oil + fish oil diet (FD) from gestating to lactating period. The blood samples of lactating sows were collected. At the age of 14 days, one piglet was selected from each litter. After the blood was collected from the anterior vena cava, LPS was injected into the neck muscle. The blood was collected 5 hours after LPS injection. At 48h after LPS injection, blood and liver samples were collected.

Results: Maternal fish oil supplementation improved the health of sows by increasing the level of HDL-C and decreasing the levels of AKP and TNF- $a$ in the plasma of sow $(P<0.05)$, and induced a positive effect on litter immune status associated with a modification of both IgG in the colostrum and IL-10 in the milk $(P<0.05)$.In addition, antioxidant capacity in piglets increased by improving the level of GSH-Px and TAOC $(P<0.05)$ in the plasma of piglets 48 h after LPS challenged. Meanwhile, the expression of GSH-Px mRNA and $p$-ERK protein in the livers of piglets was inhibited $(P<0.05)$. However, in FD group, the level of $\mathrm{IL}-1 \beta$ and IL- 6 in the plasma of piglets were significantly higher before and after LPS challenged $(P<0.05)$. The expression of NF-KB mRNA and p-IKB-a protein was increased in the liver of piglets $(P<0.05)$.

Conclusion: These results indicated that the diet of sow with fish oil might decrease the oxidative stress and inflammatory response in sows and enhance the antioxidative ability in their progenies, but might increase the susceptibility to inflammatory response in progenies.

\section{Background}

Neonatal piglet survival always bothered the modern pig industry, because the imperfect immune system of neonatal piglet makes it is sensitive to resist pathogen. The liver plays a central role in the regulation of lipid metabolism and is critical for host defense against invading pathogen and tissue repair in severe infection. Nutrition during pregnancy and lactation period may have a fundamental impact on fetal immune development [1, 2]. Fish oil is rich in long chain $n-3$ polyunsaturated fatty acids (n-3 LC-PUFAs), such as 20:5n-3 (EPA) and 22:6n-3 (DHA), which are essential fatty acid for pig and exert beneficial antiinflammatory effects in animal and cell models [3]. The sow's milk were the main source of nutrient for the sucking piglets. The lactating diet of sow with $3 \sim 5 \%$ fish oil is beneficial for the growth of sucking piglets [4-6]. Therefore, maternal nutrition is closely related to the health of the progeny.

Weaning is abruptly stressful in the neonates' life, and that stress can result in growth retardation and susceptibility to diseases in mammals. Weaning stress increased oxidative stress and transaminase in the liver of piglets and induced apoptosis, which may be related to MAPK signaling pathway activated by weaning piglets [7]. Recent evidences from animal models indicate that LPS-induced acute liver injury is caused by a variety of factors including inflammation, oxidative stress and lipid metabolism disorders. In our previous study, the supplementation of fish oil in sow diet was associated with longer gestation, 
alleviate oxidative stress in sows on farrowing day and modulate inflammatory response in sows and their offspring[8]. As mentioned above, we hypothesized that the maternal diet with fish oil could change the inflammatory response of the sows and potentiate the resistance for inflammation in the piglets suckling fish oil supplemented sow under the stimulation of immune stress.

The objective of this study was to investigate that the effects the maternal diet with fish oil on the oxidative stress and inflammatory response of sows, as well as on the oxidative stress and inflammatory response in their offspring before and after LPS challenge.

\section{Materials And Methods}

All experimental protocols were approved by the Animal Care and Use Committee of the shanghai Jiaotong University. The study took place at the experimental study farm of a feeding company (xinnong feed Itd).

\section{Animal, diet and animal management}

Twelve second-parity sows (hybrid Topigs 20 breed sows, Dutch Landrace $\times$ Great York) and their piglets [(Dutch Landrace $\times$ Great York $) \times$ Duroc] were used in the experiment. On day 85 of gestation, sows were equally divided into two groups, with six replicates per group and one sow per replicate. The back fat thickness of sows were measured at day 84 of gestation, and 12 sows which had similar back fat thickness (Soybean oil group: $15.50 \pm 0.61$ vs Fish oil group: $14.83 \pm 0.79 \mathrm{~mm} ; P=0.52$ ) were selected for our study. The back fat thickness was measured at the level of the last rib on each side and $65 \mathrm{~mm}$ from the midline by using the digital back fat indicator (BQT-521, Renco Lean-meater, USA).

From the $84^{\text {th }}$ day of gestation until the $16^{\text {th }}$ day of lactation, all 12 sows were divided into two dietary treatment groups: the first group was fed the soybean oil maternal diet (SD) and the second group was fed the fish oil supplemented diet (FD) during the experimental period. Diets were formulated according to the sow's nutrient requirements from National Research Council (NRC, 2012) [9]. For gestation diet, the SD was composed of $3 \%$ of soybean oil to make the n-6: n-3PUFA ratio 8.8:1, while the FD was composed of $0.5 \%$ of soybean oil $+2.5 \%$ of fish oil to make the n-6: n-3PUFA ratio $1.6: 1$; For lactation diet, the SD was composed of $3.5 \%$ of soybean oil to make the n-6: n-3PUFA ratio 10:1, while the FD was composed of $0.7 \%$ of soybean oil $+2.8 \%$ of fish oil to make $n-6$ : $n$-3PUFA ratio $2: 1$. Diet formulations are shown in Supplementary Table 1. The fatty acid composition in diets was determined as described by Raes et al. [10],and the fatty acid composition for experimental diets was shown in Table 1.All diets were mash feed and were stored in vacuum dark storage bags per $20 \mathrm{~kg}$, and kept in $24-28^{\circ} \mathrm{C}$ constant temperature warehouse before using.

From the $85^{\text {th }}$ day to the $109^{\text {th }}$ day of gestation, all pregnant sows were housed individually in gestation crates $(2.1 \times 0.65 \mathrm{~m})$ and were fed gestation diet. The gestational sows were fed with $3.0 \mathrm{~kg}(3.0 \mathrm{~kg} / \mathrm{day}$ diet was limited for sows during the late gestation period, so sows can eat up diets for each day ) and 
supplied twice per day (06:00 and 13:00)from the $84^{\text {th }}$ day of gestation to 5 day before farrowing. From the $110^{\text {th }}$ day of gestation to farrowing day, feed allowance was decreased by $0.5 \mathrm{~kg} / \mathrm{day}$ until no feed was supplied. On the $2^{\text {nd }}$ day after farrowing, the sows were supplied diets 3 times per day $(06: 00,13: 00$ and $18: 00$ ) with $0.75 \mathrm{~kg} /$ day initially, and diet was then increased gradually by $0.75 \mathrm{~kg} /$ day until reaching ad libitum. The room temperature of the gestation and farrowing units was approximately $24-28^{\circ} \mathrm{C}$.

Sows freely got access to water during the entire experiment.

All suckling piglets also were housed in corresponding farrowing unit with incubator and heat lamp for piglets from farrowing day to weaning day. During $48 \mathrm{~h}$ post-farrowing, the litter size was equalized to achieve 10-13 pigs by the means of cross-fostering within the same treatment group according to the number of effective nipples in each sow. Piglets were not fed the standard creep feed before $16 \mathrm{~d}$. Piglets freely got access to water.

\section{LPS treatment on piglets}

On the $14^{\text {th }}$ day after birth, twelve piglets ( 3 males and females in the SD group and the FD group) were selected in the study. One piglet per litter was selected and the average body weight is similar between the SD group and the FD group (SD: $4.31 \pm 0.04$ vs FD: $4.18 \pm 0.09 \mathrm{~kg} ; P=0.23)$. Blood sample $(5 \mathrm{~mL})$ were collected from each selected piglet, and then all selected piglets were administrated the cervical side behind the left ear with E. coli LPS at $80 \mu \mathrm{g} / \mathrm{kg}$ BW. The LPS (Escherichia coli serotype 055: B5, Sigma Chemical, St. Louis, MO 63103, USA) was dissolved in sterile $0.9 \% \mathrm{NaCl}$ solution (500 mg LPS per liter of saline). Blood samples $(5 \mathrm{~mL})$ were collected from each pig at $5 \mathrm{~h}$ and $48 \mathrm{~h}$ post-LPS challenge. All piglets were slaughter at $48 \mathrm{~h}$ post-LPS challenge.The internal organs (intestine, liver, kidney, spleen, heart and pancreas) of the piglets obtained immediately after slaughtering and measured to calculate the relative organ weight and length to the body weight.

\section{Blood and tissue sample collection}

Blood samples. Blood samples from the sows were collected from the auricular vein. Each piglet was anaesthetized with an intramuscular neck injection of pentobarbital sodium ( $35 \mathrm{mg} / \mathrm{kg} \mathrm{BW}$ ) and blood sample was then collected from the front cavity vein of each piglet. All blood samples were kept in heparinized tubes and centrifuged at $2550 \times \mathrm{g}$ for $10 \mathrm{~min}$ at $4^{\circ} \mathrm{C}$. The supernatant fraction was divided and stored at $-20^{\circ} \mathrm{C}$ for subsequent analysis.

Liver samples of piglets. The posterior half of liver samples were obtained immediately after slaughtering. The livers were washed in physiological saline, collected into $5 \mathrm{ml}$ freezing tubes, frozen in liquid $\mathrm{N}_{2}$, and then stored at $-80^{\circ} \mathrm{C}$.

\section{Analysis of oxidant and antioxidant content}


The analysis of malondialdehyde (MDA) (Cat No. A003-1-2), total superoxide dismutase (T-SOD) (Cat No. A001-1-2), glutathione peroxidase (GSH-Px) (Cat No. A005-1-2) and total antioxidant capacity (T-AOC) (Cat No. A015-3-1) in plasma was determined according to the manufacturer's instructions (Nanjing Jiancheng Bioengineering Institute, Nanjing, China). All absorbance levels were determined with a microplate reader (Synergy 2, BioTek, USA).

\section{Analysis of cytokines in Plasma}

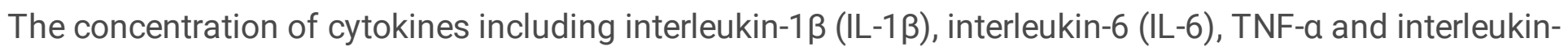
10 (IL-10) was determined using porcine-specific ELISA kits (Nanjing Jiancheng Bioengineering Institute, Nanjing, China) according to the manufacturer's instructions. Absorbance values were read in a 96-well plate reader (Synergy 2, BioTek, USA) at $450 \mathrm{~nm}$. A four parameter logistic curve-fit was generated using ELISA Calc software v0.1 (Comple-Software. lowa City, IA). The concentration of cytokines in the plasma was calculated by comparison with a standard curve.

\section{Quantitative real-time PCR}

Total RNA was isolated from liver samples using Total RNA Kit $₫$ (50) (Cat no. R6834-01; OMEGA, USA). RNA quality was verified by both agarose gel (1\%) electrophoresis and spectrometry (A260/A280, Beckman DU-800; Beckman Coulter, Inc.). One $\mu \mathrm{g}$ of RNA was reverse transcribed using the Primescript ${ }^{\mathrm{TM}}$ RT Reagent Kit with gDNA Eraser (Perfect Real Time) (RR047A, TaKaRa, Japan). Primers for all target genes are shown in Supplementary Table 2. Quantitative real-time PCR (RT-qPCR) was used to determine the relative expression level of target genes using the one-step SYBR ${ }^{\circledR}$ Premix Ex Taq (TLi RNaseH Plus) (RR420A; TaKaRa, Japan). Briefly, the final volume of the reaction mixtures $(20 \mu \mathrm{L})$ contained $10 \mu \mathrm{L}$ of SYBR Premix Ex Taq (Tli RNaseH Plus), $0.8 \mu \mathrm{L}$ of the primer pair, $0.4 \mu \mathrm{L}$ of ROX Reference Dye $\varangle, 2 \mu \mathrm{L}$ of cDNA and $6.8 \mu \mathrm{L}$ of sterile water. $\beta$-actin was used as the endogenous control gene to normalize the expression of target genes. The relative quantification of gene amplification by RT-qPCR was performed using the value of the threshold cycle $\left(C_{\mathrm{t}}\right)$. The comparative $C_{\mathrm{t}}$ value method using the formula $2^{-\Delta \triangle C \mathrm{t}}$ was employed to quantify the expression levels of target genes relative to those of $\beta$-actin using the following formula:

$2^{-\Delta \Delta C t}\left(\Delta \Delta C_{\mathrm{t}}=\left(C_{\mathrm{ttarget} g e n e}-C_{\mathrm{t} \beta \text {-actin }}\right)\right.$ treatment $-\left(C_{\mathrm{t} \text { target gene }}-C_{\mathrm{t} \beta \text {-actin }}\right)$ control $\mathbb{[}[11]$

\section{Western Blot Analysis}

The proteins in liver samples were extracted and mixed with loading buffer as previously described by Luo et al. [7]. Forty $\mu \mathrm{g}$ of proteins were separated on 10\% SDS-PAGE gels and electro-transferred to polyvinylidene difluoride (PVDF) membranes $(0.45 \mu \mathrm{m}$ pore size, IPVH00010, Millipore, MA). The membranes were blocked for $2 \mathrm{~h}$ with 5\% (w/v) skimmed milk powder (Cat No.D8340, Solarbio, Shanghai, 
China) in tris-buffered saline-tween (TBS-T) [0.5M NaCl (S7653, Sigma-Aldrich, Shanghai, China), 20mM Tris (Amresco, Shanghai, China), pH 7.5, and 0.1\% (v/v) Tween-20 (P7949, Sigma-Aldrich, Shanghai, China)], then washed three times with TBS-T, and incubated overnight at $4{ }^{\circ} \mathrm{C}$ with primary antibodies following dilutions in $5 \%$ skimmed milk powder or BSA (Cat No.0218054950, ChromatoPur ${ }^{\mathrm{TM}}$, New Zealand).The primary antibodies were anti-JNK (1:200, sc-571, Santa Cruz, USA), anti-p-JNK(1:500, orb10951, Biorbyt Ltd, UK)『anti-p38a (1:2000, sc-535, SantaCruz, USA), anti-p-p38 (1:200, sc-7973, Santa Cruz, USA), anti-ERK1/2 (1:1000, number 9102, Cell Signaling Technology, USA), anti-phospho-ERK1/2 (1:2000, number 4370, Cell Signaling Technology, USA), anti-IKBa(1:1000, number4814, Cell Signaling Technology, USA) and anti-p-IKBa (1:1000, number 9246, Cell Signaling Technology, USA) antibodies. After washing three times with TBS-T, the membranes were then incubated with goat anti-rabbit (1:10000, ab97051, Abcam, UK) or goat anti-mouse IgG-HRP (1:2000, sc-2005, Santa Cruz, USA) antibodies for $2 \mathrm{~h}$. Afterwards, blots were developed using Amersham ${ }^{\mathrm{TM}}$ ECLTM Prime Western Blotting Detection Reagent (Catalogue No.RPN2232, Healthcare UK limited little Chalfont). Image acquisition was performed on an enhanced chemiluminescence detection system (Tanon, Shanghai, China). Image J software was used to quantify the density of the specific protein bands.

\section{Statistical analyses}

All variables were tested for normal distribution by Shapiro-Wilk test. Individual sow or piglet was the experimental unit for the indices. The data were analysed by using the procedure of $t$-test (IBM SPSS Statistics 20).Because of the differences among treatments at the start of piglet study, plasma TG,IL$1 \beta, I L-6$ and IL-10 concentrations of piglet before LPS challenged were regarded as a covariate. Results were expressed as means and the standard error of the mean (SEM). A P-value less than 0.05 was considered to be statistically significant.

\section{Results}

\section{Organ indices of piglets}

The organ indices of piglets were shown in Table 2. There were no differences in the relative intestinal length of piglets between the SD group and the FD group. The FD had no effect on the relative liver weight, pancreas weight, brain weight, spleen weight and kidney weight of piglets.

\section{Effect of fish oil maternal diet on the lipid profile and liver function of sows}

The FD treatment had significant increased plasma level of HDL-C compared with the SD treatment $(P<$ 0.05) (Table 3). There were no difference between the two groups for the level of TG, T-CHO and LDL-C in the plasma of sow. The level of AKP in the plasma of sow was lower in the FD group relative to the SD 
group on the 16 day of lactation $(P<0.05)$. The FD had no significant influence on the level of AST and ALT in the plasma of sows.

\section{Effect of maternal diet with fish oil on the oxidative stress parameters and immune cytokines in the plasma of sows}

The FD decreased the concentration of TNF-a $(P<0.05)$, but had no significant influence on the concentration of IL-1 $1 \beta, \mathrm{IL}-6$ and IL-10 in the plasma of sow on the 16 day of lactation (Table 4). Moreover, The FD had no significant influence on the 8-iso-PG, T-SOD, GSH-Px and T-AOC in sow plasma on the 16 day of lactation $(P>0.05)$.

\section{Effect of the maternal diet with fish oil on the content of chemical composition, immunoglobulins and immune cytokines in the colostrum and milk}

The FD significantly increased the content of IgG in the colostrum, but had no significant influence on the content of $\beta$-casein, slgA and IgM in the colostrum and milk (Table 5). The concentration of TNF- $\alpha$ in the milk of the FD group was lower than that of the SD group $(P<0.05)$, while the concentration of IL-10 in the milk of the FD group was higher than that in the SD group $(P<0.05)$. The FD had no effect on the IL-1 $\beta$, IL6 , TNF- $a$ and IL-10 in the colostrum.

\section{Effect of the maternal diet with fish oil on the lipid profile and liver function in the plasma of piglets pre-/post-LPS challenge}

The FD group had significantly decreased plasma level of TG compared with the SD group on the 14 day of suckling piglets before LPS challenged $(P<0.05)$, but had no effect on the level of T-CHO, LDL-C and HDL-C in the plasma of piglets pre-LPS challenge. The FD had no significant influence on the level of AST, ALT and AKP in the plasma of the suckling piglets pre-LPS challenge.

The level of T-CHO and LDL-C in the plasma of the suckling piglets from the FD group was lower than that from SD group at 5 h post-LPS challenge $(P<0.05)$. Conversely, the level of ALT and AKP increased in the FD group relative to the SD group in the plasma of the piglets at $48 \mathrm{~h}$ post-LPS challenge $(P<0.05)$.

\section{Effect of the maternal diet with fish oil on the oxidative stress status in piglets}

The activities of GSH-Px and T-AOC in the plasma of suckling piglets were higher in the FD group than that in the SD group at $48 \mathrm{~h}$ post-LPS challenge $(P<0.05)$ (Fig. 1c, $1 \mathrm{~d}$, respectively), while the FD had no effect on the 8-iso-PG and T-SOD in the plasma of suckling piglets pre-/post-LPS challenge $(P>0.05)$ 
(Fig. 1a, 1b,respectively). Conversely, the relative expression of GSH-Px mRNA in the livers of piglets of the FD group was lower than that of the SD group at 48h post-LPS challenge $(P<0.05)$ (Fig. $1 \mathrm{e})$.

\section{Effect of the maternal diet with fish oil on inflammatory response in piglets}

The FD increased the concentration of IL-1 $\beta$, IL- 6 and IL-10 in the plasma of piglets pre-LPS challenge $(P<0.05)$ (Fig. 2b, 2c, 2d, respectively), while the FD had no effect on the concentration of TNF-a in the plasma of piglets pre-/post-LPS challenge. The concentration of IL- 6 in the plasma of piglets was higher in the FD group than that in the SD group at 48 h post-LPS challenge $(P<0.05)$ (Fig.2c). The concentration of IL-1 $\beta$ in the plasma of piglets was significantly higher in the FD group than that in the SD group at both $5 \mathrm{~h}$ and $48 \mathrm{~h}$ post-LPS challenge $(P<0.05)$ (Fig.2b). Meanwhile, the relative expression of IL-1 $\beta$ mRNA in the livers of piglets was also higher in the FD group than that in the SD group at $48 \mathrm{~h}$ post-LPS challenge $(P<0.05)$ (Fig.2f). There is no diet effect on the relative expression of IL-6 and IL-10 mRNA in the livers of piglets at $48 \mathrm{~h}$ post-LPS challenge.

\section{Effect of the maternal diet with fish oil on mechanism parameters in piglets}

The relative expression of G-protein coupled receptor 120 (GPR120) the livers of piglets was lower in the FD group than that in the SD group at 48 h post-LPS challenge $(P<0.05)$ (Fig. 3a). Conversely, the relative expression of TGF-beta activated kinase 1 (TAK1) and NFKB mRNA in the livers of piglets was higher in the FD group than that in the SD group at 48h post-LPS challenge $(P<0.05)$ (Fig. 3a).

Fish oil supplementation in maternal diet significantly down-regulated the $\mathrm{p}$-ERK/ERK protein in the livers of piglets at $48 \mathrm{~h}$ post-LPS challenge $(P<0.05)$ (Fig. 3b). Conversely, the expression of $\mathrm{p}-\mathrm{I} \mathrm{KBa} / \mathrm{IkBa}$ proteins in the livers of piglets was significantly up-regulated in the FD group at $48 \mathrm{~h}$ post-LPS challenge $(P<0.05)$ (Fig. 3b).

\section{Discussion}

In this study, we investigated that the effects maternal diet with fish oil on the oxidative stress and inflammatory response of lactating sows, as well as the effects on oxidative stress and inflammatory response of their offspring before and after LPS stimulation.

The supplementation of fish oil in the diet of perinatal sows may help to reduce the inflammatory response and improve the liver function of lactating sows. Our results showed that maternal diet with fish oil could increase the plasma HDL-C level and decrease the plasma AKP and TNF-a levels, but had no significant effect on the markers of oxidative stress and antioxidant enzymes in the plasma of sow. These results were consistent with the report of Papadopoulos et al. (2009), which reported that fish oil 
diet significantly decreased the levels of IL- 6 and TNF-a in the serum of sow on the $3^{\text {rd }}$ and $8^{\text {th }}$ day of lactation [12]. Tanghe et al. (2013) reported that the perinatal sows' diet with fish oil had no effect on the oxidative stress state of sows[13]. However, the inconsistent reports found that dietary fish oil had no effect on the expression of genes related to cholesterol synthesis and absorption in the liver of lactation sows, but had minor effect on the hepatic lipid metabolism which had no effect on the milk production of sows [14]. Shen et al. (2015) suggested that the supplementation of olive oil in the maternal diets significantly reduced IL-1 $\beta$ and TNF- $\alpha$ in the milk, but increased the oxidative stress of sows in fish oil group [15]. The reasons for these inconsistency may be associated with the experimental design, diet storage (vacuum dark bag storage or common bag storage) and the dietary antioxidant supply[16].

Dietary supplementation with fish oil to sows induced a positive effect on litter immune status associated with a modification of both cytokines and immunoglobulins (IgG and IgM) in milk [17]. Dietary fish oil resulted in an increase in antibodies in both blood and colostrum from sows after vaccination, together with an increase in antibodies, leukocyte and IgG in the blood of piglet[18]. n-3PUFA supplementation could reduce the inflammatory stimulation of LPS on mammary gland and the levels of IL-6, IL-8, IL-1 $\beta$ and TNF-a in milk $[15,19]$. Our results showed that the level of IgG and IL-10 in colostrum were increased and TNF-a in normal milk were decreased by adding fish oil to the diets of gestating and lactating sows.

In our study, at the age of 14 days, the TG level in the plasma of sucking piglets was reduced, but the levels of ALT,AKP,IL-1 $\beta, I L-6$ and IL-10 in the plasma of sucking piglets were increased in the FD group. ALT and AKP is positively correlated with the degree of abnormal liver tissue [20-22]. IL-10 is an antiinflammatory regulatory cytokine, which could induce antibody production and improve antiinflammatory ability [23]. IL-10 in milk can cross the intestinal barrier and affect thymic development in progeny [24].The increase of IL-10 in plasma of sucking piglets might be transfer from milk to piglets. However, the increase of IL-1 $\beta$ and IL- 6 in the plasma of piglets suggested the increase of inflammatory response in the FD group. This is inconsistent with the reports of Leonard et al. (2010) and McAfee et al. (2019), which found that providing fish oil diet to sows beginning 5-7 days before delivery and during lactation can increase leukocyte and lymphocyte phagocytosis in piglets at weaning, and reduce the acute physiological stress response in the pigs postweaning by attenuating the release of inflammatory cytokines (IL-1 $\beta$, IL-6, and TNF- $\alpha$ ) $[25,26]$. The reason for this inconsistency may be related to the feeding time period and the dose of fish oil. In the previous studies, the intake of fish oil for sows is very limited before delivery, because the feeding allowance of sows was gradually decreased from 5-7 days before delivery in actual pig production. So far, the experimental research about fish oil supplementation for more than 4 weeks were limited. Luo et al. (2013) reported that the diet of sow with fish oil from 10 days before delivery can reduce the expression of inflammatory cytokines in skeletal muscle and promote the growth of suckling piglets, but increase the expression of inflammatory cytokines in the spleens of weaned piglets by continuously feeding fish oil diet to weaned piglets [27].

Maternal fish oil supplementation potentiated antioxidant capacity in suckled piglets. Our results showed that there were no difference on the oxidative stress state in the piglets suckling fish oil supplemented sows before LPS challenged. However, 48 hours after LPS challenged, the levels of GSH-Px and T-AOC in 
plasma of piglet were increased in FD group, while the oxidative stress marker products had no effect. Marianne et al. (2018) reported that supplementation of DHA in piglets can significantly reduce the oxidative stress response caused by lipid peroxidation[28]. Although there is no report about the effect of dietary fish oil on the mRNA expression of antioxidant enzyme in the livers of piglets, placental antioxidant system has a proper capability to compensate for the oxidative stress through increasing gene expression of antioxidant enzymes in placenta $[8,29,30]$. We postulated that hepatic antioxidant system of piglets may also have an adaptive response to oxidative stress. In addition, oxidative stress can induce ERK phosphorylation in hepatocytes, but DHA can regulate the activation of ERK $1 / 2$ in MAPK $[31,32]$. The cytokines produced by inflammatory stimulation could cause a secondary attack on the tissue by inducing ROS production, but DHA can directly reduce ROS produced by cytokines through inhibiting the activation of ERK1/2 [32] . In our study, the expression of GSH-Px mRNA and the phosphorylation of ERK protein in the liver of piglets were decreased in piglets suckling fish oil supplemented sows.

Interestingly, our results were inconsistent that the effects of fish oil supplementation on inflammatory response and oxidative stress in piglets suckling fish oil supplemented sows. The initial phase of the acute inflammatory response is characterized by the production of pro-inflammatory mediators followed by a second phase in which lipid mediators with pro-resolution activities may be generated[33]. Dysregulation of the dynamic inflammatory process will directly lead to tissue damage. LXA4, is an eicosanoid generated, which is one of the endogenous local mediators of resolution during acute inflammatory responses. NF-KB activation associated with both the onset and the resolution of inflammation in both rat carrageenin pleurisy and mouse carrageenan air pouch[34]. NF-KB DNA binding activity further increased at 24 and $48 \mathrm{~h}$ after inflammatory stimulation in the presence of IKBa protein[34]. Myeloid cells represent a major target for LXA4 and reflect the attenuation of NF-KB activity [35-37]. Therefore, n-6 PUFA participates in the resolution of inflammation [38]. In addition, the production of LXA4 requires the function of ALOX15 enzyme. When the same amount of n-3PUFA and n-6PUFA substrates exist, the enzyme shows the priority of using DHA and EPA instead of 20:4n-6 [39]. Our previous study reported that the total proportion of n-3PUFA in the livers of piglets suckling fish oil supplemented sows were higher than that of n-6 PUFA[40]. In our present study, the relative expression of NF-KB mRNA and the expression of $\mathrm{p}-\mathrm{I} \mathrm{KBa} / \mathrm{lkBa}$ proteins in the livers of piglets after $48 \mathrm{~h}$ LPS challenge were decreased. These results indicated that the continuous supplementation of fish oil for more than 4 weeks may affect the process of resolution of inflammation in the liver of piglets and lead to liver injury.

\section{Conclusion}

In summary, the diet of sow with fish oil might decrease the oxidative stress and inflammatory response in sows and enhance the antioxidative ability in their progenies, but might increase the susceptibility to inflammatory response in progenies.

\section{Abbreviations}


FD: Fish oil diet; SD: Soybean oil diet; T-SOD: Total superoxide dismutase; GSH-Px: Glutathione peroxidase; T-AOC: Total antioxidant capacity; 8-iso-PG $₫ 8$-iso-prostaglandin; ALT: Glutamic oxaloacetylase; AST: glutamic-pyruvic transaminase; AKP: alkaline phosphatase; TG: triglyceride; T-CHO: total cholesterol; LDL-C: low-density lipoprotein cholesterol; HDL-C: high-density lipoprotein cholesterol; IL1及:Interleukin-1 $\beta$; IL-6:Interleukin-6;TNF-a:Tumor necrosis factor a; IL-10: Interleukin-10;TAB1:TAK1 binding protein 1; TAK1:Transforming growth factor- $\beta$ activated kinase 1;PTGS2:Prostaglandinendoperoxide synthase 2;ALOX5:lipoxygenase enzyme 5; GPR120:G-protein coupled receptor 120;TLR4:Toll-like receptor 4;NF-KB:Nuclear factor-B; PPARY :Peroxisome proliferator activated receptor gamm

\section{Declarations}

\section{Acknowledgements}

We thanks Xinnong Feed Co. Ltd for supplying pig experiment farm ,feed process and people to care of the pigs. The author would like to thank Dr. M.Y.Y for technical help.

\section{Funding}

This work was supported by The National Natural Science Foundation of China (No. 31872367) for financially supported.

\section{Availability of data and materials}

The data used to support the findings of this study are included within the article and the supplementary information file.

\section{Authors' contributions}

WL and JX designed the study, WL and WN performed the research, WL, JZ and WX collected the data, WL analyzed the data, WL wrote the manuscript. All authors read and approved the final manuscript.

\section{Competing interests}

The authors disclose that there is no conflict of interest.

\section{Consent for publication}

Not applicable. 


\section{Ethics approval}

The protocol used in this experiment approved by Shanghai Jiao Tong University Institutional Animal Care and Use Committee and was performed in accordance with the National Research Council's Guide for the Care and Use of Laboratory Animals.

\section{Reference}

1. Prescott, S.L. and J.A. Dunstan, Prenatal Fatty Acid Status and Immune Development: The Pathways and the Evidence. Lipids, 2007. 42(9): p. 801.

2. Calder, P.C., et al., Is there a role for fatty acids in early life programming of the immune system? Proc Nutr Soc, 2010. 69(3): p. 373-380.

3. Calder, P.C. and R.F. Grimble, Polyunsaturated fatty acids, inflammation and immunity. Eur J Clin Nutr, 2002. 56(3): p. S14-S19.

4. Schellingerhout, A., H. Everts, and A. Beynen, Influence of dietary n-3 polyunsaturated acids, in the form of either linseed or fish oil, on growth performance, small intestinal morphology and essential fatty acid status of weanling piglets. 2020.

5. Rooke, J.A., M. Shanks, and S.A. Edwards, Effect of offering maize, linseed or tuna oils throughout pregnancy and lactation on sow and piglet tissue composition and piglet performance. Animal science, 2000. 71(2): p. 289-299.

6. Gaines, A.M., et al., Effect of menhaden fish oil supplementation and lipopolysaccharide exposure on nursery pigs: II. Effects on the immune axis when fed simple or complex diets containing no spraydried plasma. Domest Anim Endocrinol, 2003. 24(4): p. 353-365.

7. Luo, Z., et al., Weaning Induced Hepatic Oxidative Stress, Apoptosis, and Aminotransferases through MAPK Signaling Pathways in Piglets. Oxid Med Cell Longev, 2016. 2016: p. 4768541.

8. Luo, W.L., et al., The Effect of Maternal Diet with Fish Oil on Oxidative Stress and Inflammatory Response in Sow and New-Born Piglets. Oxid Med Cell Longev, 2019. 2019: p. 6765803.

9. NRC, ed. Nutrient requirements of swine. 11th revised edition ed. National Research Council (NRC)2012, National Academies Press: Washington, DC, USA.

10. Raes, K., S.d. Smet, and D. Demeyer, Effect of double-muscling in Belgian Blue young bulls on the intramuscular fatty acid composition with emphasis on conjugated linoleic acid and polyunsaturated fatty acids. Animal Science, 2001. 73(02): p. 253-260.

11. Livak, K.J. and T.D. Schmittgen, Analysis of relative gene expression data using real-time quantitative PCR and the 2(-Delta Delta C(T)) Method. Methods, 2001. 25(4): p. 402-8.

12. Papadopoulos, G.A., et al., Peripartal feeding strategy with different n-6: n-3 ratios in sows: effects on sows' performance, inflammatory and periparturient metabolic parameters. Br J Nutr, 2009. 101(3): p. 348-57. 
13. Tanghe, S., S. Millet, and S. De Smet, Echium oil and linseed oil as alternatives for fish oil in the maternal diet: Blood fatty acid profiles and oxidative status of sows and piglets. J Anim Sci, 2013. 91(7): p. 3253-3264.

14. Gessner, D.K., et al., Effect of dietary fish oil on the expression of genes involved in lipid metabolism in liver and skeletal muscle of lactating sows. J Anim Physiol Anim Nutr (Berl), 2016. 100(2): p. 33747.

15. Shen, Y., et al., Fish Oil and Olive Oil Supplementation in Late Pregnancy and Lactation Differentially Affect Oxidative Stress and Inflammation in Sows and Piglets. Lipids, 2015. 50(7): p. 647-58.

16. DM, F.-D., Impact of oxidized corn oil and synthetic antioxidant on swine performance, antioxidant status of tissues, pork quality and shelf life evaluation, in Philosophy in Animal Sciences2009, University of Illinois at Urbana-Champaign.

17. Jin, C., et al., Influence of dietary fat source on sow and litter performance, colostrum and milk fatty acid profile in late gestation and lactation. Animal Science Journal, 2017. 88(11): p. 1768-1778.

18. Mitre, R., et al., Humoral defence improvement and haematopoiesis stimulation in sows and offspring by oral supply of shark-liver oil to mothers during gestation and lactation. Br J Nutr, 2005. 94(5): p. 753-762.

19. Lin, S., et al., Mammary inflammatory gene expression was associated with reproductive stage and regulated by docosahexenoic acid: in vitro and in vivo studies. Lipids Health Dis, 2016. 15(1): p. 215.

20. Panelli, M., et al., Bark of Passiflora edulis Treatment Stimulates Antioxidant Capacity, and Reduces Dyslipidemia and Body Fat in $\mathrm{db} / \mathrm{db}$ Mice. 2018. 7.

21. Al Zarzour, R.H., et al., Phyllanthus Niruri Standardized Extract Alleviates the Progression of NonAlcoholic Fatty Liver Disease and Decreases Atherosclerotic Risk in Sprague-Dawley Rats. Nutrients, 2017. 9(7).

22. Li X, C.Y., Liu Z, Chen H, Mao H. Coll The Relationship Between Liver Injury and Serum Levels of CReactive Protein and Procalcitonin in Patients With Acute Pancreatitis J Coll Physicians Surg Pak 2019. $29((3)):$ p. 287-289.

23. Castellote, C., et al., Premature Delivery Influences the Immunological Composition of Colostrum and Transitional and Mature Human Milk. The Journal of Nutrition, 2011. 141(6): p. 1181-1187.

24. Ballard, O. and A.L. Morrow, Human milk composition: nutrients and bioactive factors. Pediatr Clin North Am, 2013. 60(1): p. 49-74.

25. McAfee, J.M., et al., Effect of omega-3 polyunsaturated fatty acid (n-3 PUFA) supplementation to lactating sows on growth and indicators of stress in the postweaned pig12. J Anim Sci, 2019. 97(11): p. 4453-4463.

26. Leonard, S.G., et al., Effect of maternal fish oil and seaweed extract supplementation on colostrum and milk composition, humoral immune response, and performance of suckled piglets1. J Anim Sci, 2010. 88(9): p. 2988-2997.

27. Luo, J., et al., Responses of growth performance and proinflammatory cytokines expression to fish oil supplementation in lactation sows' and/or weaned piglets' diets. Biomed Res Int, 2013. 2013: p. 
905918.

28. Marianne, H., et al., DHA reduces oxidative stress following hypoxia-ischemia in newborn piglets: A study of lipid peroxidation products in urine and plasma. J Perinat Med, 2017. 46.

29. Lappas, M., A. Mitton, and M. Permezel, In response to oxidative stress, the expression of inflammatory cytokines and antioxidant enzymes are impaired in placenta, but not adipose tissue, of women with gestational diabetes. J Endocrinol, 2010. 204(1): p. 75-84.

30. Su, G., et al., Effects of oil quality and antioxidant supplementation on sow performance, milk composition and oxidative status in serum and placenta. Lipids Health Dis, 2017. 16(1): p. 107.

31. McCubrey, J.A., M.M. LaHair, and R.A. Franklin, Reactive Oxygen Species-Induced Activation of the MAP Kinase Signaling Pathways. Antioxidants \& Redox Signaling, 2006. 8(9-10): p. 1775-1789.

32. Massaro, M., et al., The omega-3 fatty acid docosahexaenoate attenuates endothelial cyclooxygenase-2 induction through both $\operatorname{NADP}(\mathrm{H})$ oxidase and PKC epsilon inhibition. Proc Natl Acad Sci U S A, 2006. 103(41): p. 15184-15189.

33. Maderna, P. and C. Godson, Lipoxins: resolutionary road. Br J Pharmacol, 2009. 158(4): p. 947-59.

34. Lawrence, T., et al., Possible new role for NF-kB in the resolution of inflammation. Nat Med, 2001. 7(12): p. 1291-1297.

35. Buckley, Christopher D., Derek W. Gilroy, and Charles N. Serhan, Proresolving Lipid Mediators and Mechanisms in the Resolution of Acute Inflammation. Immunity, 2014. 40(3): p. 315-327.

36. Crean, D. and C. Godson, Specialised lipid mediators and their targets. Semin Immunol, 2015. 27(3): p. 169-76.

37. Brennan, E.P., et al., Lipoxins Protect Against Inflammation in Diabetes-Associated Atherosclerosis. Diabetes, 2018. 67(12): p. 2657.

38. Gutierrez, S., S.L. Svahn, and M.E. Johansson, Effects of Omega-3 Fatty Acids on Immune Cells. Int J Mol Sci, 2019. 20(20).

39. Kutzner, L., et al., Mammalian ALOX15 orthologs exhibit pronounced dual positional specificity with docosahexaenoic acid. Biochimica et Biophysica Acta (BBA) - Molecular and Cell Biology of Lipids, 2017. 1862(7): p. 666-675.

40. Luo, W., et al., Effect of fish oil supplementation in sow diet during late gestation and lactation period on litter characteristics, milk composition and fatty acid profile of sows and their offspring. Italian Journal of Animal Science, 2019. 19(1): p. 8-17.

\section{Tables}

Table 1. Fatty acid composition of the diets. 


\begin{tabular}{|c|c|c|c|c|}
\hline & \multicolumn{2}{|c|}{ Gestation diet (\%) } & \multicolumn{2}{|c|}{ Lactation diet (\%) } \\
\hline & $\mathrm{SD}$ & FD & SD & FD \\
\hline \multicolumn{5}{|c|}{ Fatty acids (g/100 g total fatty acids) } \\
\hline $14: 0$ & 0.29 & 3.75 & 0.12 & 3.15 \\
\hline $16: 0$ & 15.39 & 19.16 & 14.35 & 17.87 \\
\hline $16: 1$ & n.d & 4.79 & 0.20 & 4.23 \\
\hline $18: 0$ & 3.21 & 2.74 & 3.55 & 3.08 \\
\hline $18: 1$ & 21.33 & 17.60 & 24.10 & 19.72 \\
\hline $18: 2 n-6$ & 53.73 & 29.96 & 52.49 & 32.65 \\
\hline $18: 3 n-3$ & 6.08 & 2.56 & 5.20 & 2.62 \\
\hline $20: 0$ & n.d & 1.35 & n.d & 1.10 \\
\hline $20: 1$ & n.d & n.d & n.d & 0.73 \\
\hline $20: 5 n-3$ & n.d & 9.8 & n.d & 8.07 \\
\hline $22: 5 n-3$ & n.d & 0.87 & n.d & 0.79 \\
\hline $22: 6 n-3$ & n.d & 6.17 & n.d & 5.46 \\
\hline n-6:n-3 & 8.84 & 1.54 & 10.09 & 1.93 \\
\hline
\end{tabular}

$\mathrm{SD}=$ Soybean oil diet; $\mathrm{FD}=$ Fish oil diet; $\mathrm{n} . \mathrm{d}=$ not detectable; $\mathrm{n}-3=\mathrm{n}-3$ poly unsaturated fatty acid; $n-6=n-6$ ploy unsaturated fatty acid; $n-6: n-3=n-6$ polyunsaturated fatty acids: $n-3$ polyunsaturated fatty acids.

Table 2. Organ index of piglets. 


\begin{tabular}{|c|c|c|c|c|}
\hline & SD & FD & $P$ value & \multirow{9}{*}{$\begin{array}{l}\mathrm{SD}=\text { Soybean oil diet, } \\
\mathrm{FD}=\text { Fish oil diet } \\
\text { Values are means } \pm \text { SEM, } \mathrm{n} \\
=6 \text {, } \\
* \text { Mean values were } \\
\text { significantly different } \\
\text { between the SD group and } \\
\text { the FD group }(P<0.05) \text {. } \\
\text { NS, not significant }\end{array}$} \\
\hline Initial weight (kg) & $4.12 \pm 0.05$ & $4.11 \pm 0.03$ & 0.87 & \\
\hline Slaughter weight $(\mathrm{kg})$ & $4.33 \pm 0.03$ & $4.2 \pm 0.08$ & 0.20 & \\
\hline Weight gain $(\mathrm{g} / \mathrm{d})$ & $104.2 \pm 23.61$ & $45.5 \pm 44.47$ & 0.31 & \\
\hline Heart weight: BW & $6.51 \pm 0.24$ & $6.89 \pm 0.32$ & 0.37 & \\
\hline Lung weight: BW & $13.82 \pm 0.89$ & $13.08 \pm 0.28$ & 0.45 & \\
\hline Liver weight: BW & $31.88 \pm 2.41$ & $32.50 \pm 1.95$ & 0.85 & \\
\hline Pancreas weight: BW & $1.28 \pm 0.07$ & $1.33 \pm 0.06$ & 0.60 & \\
\hline Spleen weight: BW & $5.76 \pm 0.44$ & $6.88 \pm 0.38$ & 0.08 & \\
\hline Kidney weight: BW & $5.76 \pm 0.44$ & $6.88 \pm 0.38$ & 0.08 & \multirow{3}{*}{$\begin{array}{l}\text { Table 3. Effect of maternal } \\
\text { diet with fish oil on the lipid }\end{array}$} \\
\hline Brain weight: BW & $9.46 \pm 0.23$ & $10.15 \pm 0.26$ & 0.08 & \\
\hline Intestinal length: BW & $168.3 \pm 6.12$ & $180.9 \pm 7.11$ & 0.21 & \\
\hline
\end{tabular}

sows.

\begin{tabular}{|c|c|c|c|}
\hline & \multicolumn{3}{|c|}{ Lactation 16d } \\
\hline & SD & FD & $\overline{\text { SEM }}$ \\
\hline \multicolumn{4}{|l|}{ Lipid profile } \\
\hline $\mathrm{TG} \square \mathrm{mmol} / \mathrm{L})$ & 0.59 & 0.58 & 0.12 \\
\hline T-CHO $\square \mathrm{mmol} / \mathrm{L})$ & 1.71 & 2.14 & 0.23 \\
\hline LDL-C $\llbracket \mathrm{mmol} / \mathrm{L})$ & 1.73 & 1.57 & 0.21 \\
\hline HDL-C $\square \mathrm{mmol} / \mathrm{L})$ & 1.12 & $1.56^{*}$ & 0.12 \\
\hline \multicolumn{4}{|l|}{ Liver function } \\
\hline AST(GOT) $(\mathrm{U} / \mathrm{L})$ & 5.79 & 4.3 & 1.27 \\
\hline ALT(GPT) (U/L) & 5 & 3.36 & 1.24 \\
\hline $\mathrm{AKP}(\mathrm{U} / \mathrm{L})$ & $168.43^{*}$ & 88.85 & 26.99 \\
\hline
\end{tabular}

$\mathrm{TG}=$ triglyceride; $\mathrm{T}-\mathrm{CHO}=$ total cholesterol; LDL-C = low-density lipoprotein cholesterol; HDL-C = high-density lipoprotein cholesterol; AST = aspartate amino transferase; GPT = glutamic-pyruvic transaminase; $\mathrm{AKP}=$ alkaline phosphatase; NS=not significant.

All results are presented as mean \pm SEM $(n=6)$, SEM=pool SEM.

*Mean values were significantly different between the two groups at the same time point $P<0.05$. 
Table 4. Effect of maternal diet with fish oil on the immune cytokines and oxidative stress parameters in the plasma of sows.

\begin{tabular}{lccc}
\hline & SD & FD & SEM \\
\hline Cytokines & & & \\
IL-1 $\beta$ (ng/L) & 48.45 & 60.64 & 5.82 \\
IL-6 (ng/L) & 67.52 & 55.62 & 6.61 \\
IL-10 (ng/L) & 33.33 & 34.77 & 2.89 \\
TNF- $\alpha$ (ng/L) & $322.1 *$ & 264.34 & 13.36 \\
Oxidative stress parameters & & & \\
8-iso-PG (ng/L) & 336.77 & 324.98 & 31.12 \\
T-SOD (U/mL) & 164.18 & 169.55 & 3.14 \\
GSH-Px (U/mL) & 1091.4 & 1067.2 & 45.87 \\
T-AOC (U/mL) & 0.22 & 0.24 & 0.02 \\
\hline
\end{tabular}

$\mathrm{FD}=$ fish oil diet; $\mathrm{SD}=$ soybean oil diet; IL-1 $\beta=$ Interleukin-1 $\beta$; IL-6= Interleukin-6; IL-10= Interleukin-10; TNF- $\alpha=$ Tumor necrosis factor -alpha; 8-iso-PG=8-iso prostaglandin; $\mathrm{T}$ $\mathrm{SOD}=$ total superoxide dismutase; GSH-Px, glutathione peroxidase; T-AOC=total antioxidant capacity.

All results are presented as mean $\pm \operatorname{SEM}(n=6)$

SEM=pool SEM

*Mean values were significantly different between the two groups at the same time point $\mathrm{P}<0.05$.

Table 5. Effect of maternal diet with fish oil on the content of chemical composition, immunoglobulins and immune cytokines in the colostrum and milk. 


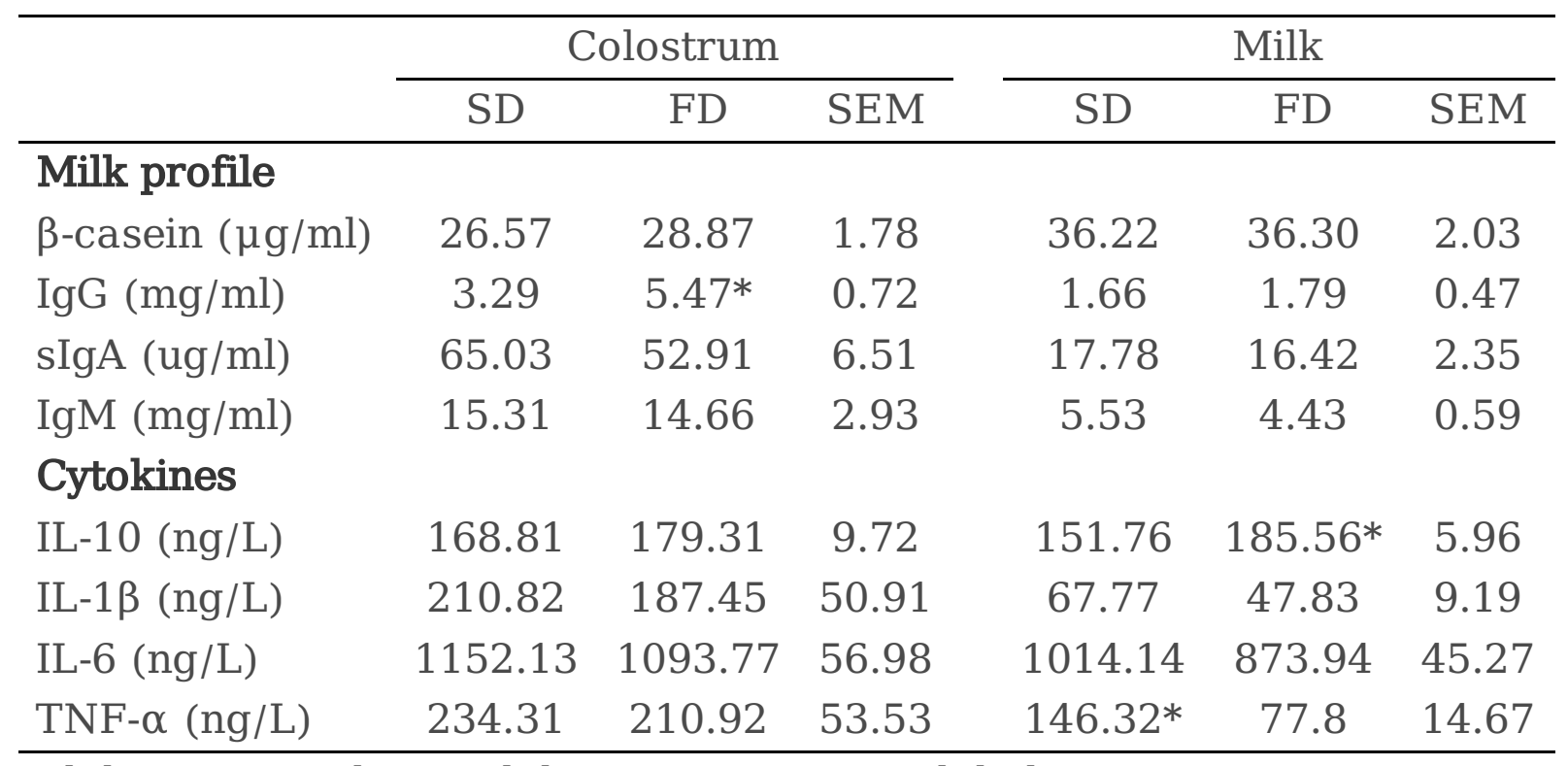

$\mathrm{FD}=$ fish oil diet; $\mathrm{SD}=$ soybean oil diet; $\mathrm{IgG}=$ immunoglobulin $\mathrm{G} ; \mathrm{sIgA}=$ secretory immunoglobulin A; IgM = immunoglobulin M; IL-1 $\beta=$ interleukin-1 $\beta$; IL-6= interleukin-6; IL$10=$ interleukin-10; TNF- $\alpha=$ tumour necrosis factor - alpha.

Colostrum results are presented as mean \pm SEM $(n=6)$; milk results are presented as mean \pm SEM $(n=4)$.

$\mathrm{SEM}=$ pool SEM

*Mean values were significantly different between the two groups at the same time point $\mathrm{P}<0.05$.

Table 6. Effect of maternal diet with fish oil on the lipid profile and liver function in the plasma of piglet pre-/post-LPS challenge. 


\begin{tabular}{|c|c|c|c|c|c|c|c|}
\hline & \multicolumn{2}{|c|}{$14 d$} & \multicolumn{2}{|c|}{$\begin{array}{c}\text { 14d+5h after LPS } \\
\text { challenge }\end{array}$} & \multicolumn{2}{|c|}{$\begin{array}{l}14 \mathrm{~d}+48 \mathrm{~h} \text { after } \\
\text { LPS challenge }\end{array}$} & \multirow[t]{2}{*}{ SEM } \\
\hline & SD & FD & SD & FD & SD & FD & \\
\hline \multicolumn{8}{|l|}{$\begin{array}{l}\text { Lipid } \\
\text { profile }\end{array}$} \\
\hline $\begin{array}{l}\text { TG } \\
(\mathrm{mmol} / \mathrm{L})\end{array}$ & $1.07 *$ & 0.68 & $0.56^{*}$ & 0.35 & 0.62 & 0.77 & 0.07 \\
\hline $\begin{array}{l}\mathrm{T}-\mathrm{CHO} \\
(\mathrm{mmol} / \mathrm{L})\end{array}$ & 6.13 & 5.19 & $4.63 *$ & 3.61 & 5.32 & 4.55 & 0.51 \\
\hline $\begin{array}{l}\text { LDL-C } \\
(\mathrm{mmol} / \mathrm{L})\end{array}$ & 3.63 & 2.6 & $3.51 *$ & 2.46 & 3.33 & 2.86 & 0.35 \\
\hline $\begin{array}{l}\text { HDL-C } \\
(\mathrm{mmol} / \mathrm{L})\end{array}$ & 3.07 & 3.68 & 1.72 & 1.59 & 2.64 & 2.85 & 0.21 \\
\hline \multicolumn{8}{|l|}{$\begin{array}{l}\text { Liver } \\
\text { function }\end{array}$} \\
\hline AST (U/L) & 6.7 & 8.28 & 17.51 & 10.45 & 6.11 & 6.4 & 1.26 \\
\hline ALT (U/L) & 8.88 & 7.83 & 15.03 & 11.93 & 9.45 & $13.2^{*}$ & 1.39 \\
\hline $\mathrm{AKP}(\mathrm{U} / \mathrm{L})$ & 959.55 & 1059.73 & 1042.55 & 1121.06 & 646.14 & $965.67 *$ & 70.92 \\
\hline
\end{tabular}

$\mathrm{FD}=$ fish oil diet; $\mathrm{SD}=$ soybean oil diet; $\mathrm{G} 84 \mathrm{~d}=$ =gestation $84 \mathrm{~d} ; \mathrm{Fd}=$ farrowing day;

L16d=lactation 16d; TG= triglyceride; $\mathrm{T}-\mathrm{CHO}=$ total cholesterol; LDL-C = low-density lipoprotein cholesterol; HDL-C = high-density lipoprotein cholesterol; AST = aspartate amino transferase; $\mathrm{GPT}=$ glutamic-pyruvic transaminase; $\mathrm{AKP}=$ alkaline phosphatase. All results are presented as mean $\pm \operatorname{SEM}(n=6)$

SEM=pool SEM

*Mean values were significantly different between the two groups at the same time point $P<0.05$.

\section{Figures}


GD FD

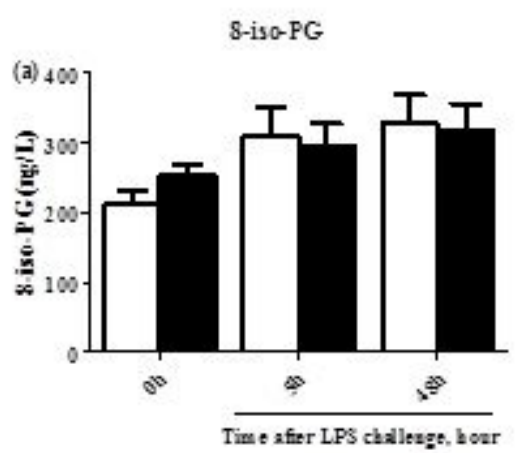

GSH-Px
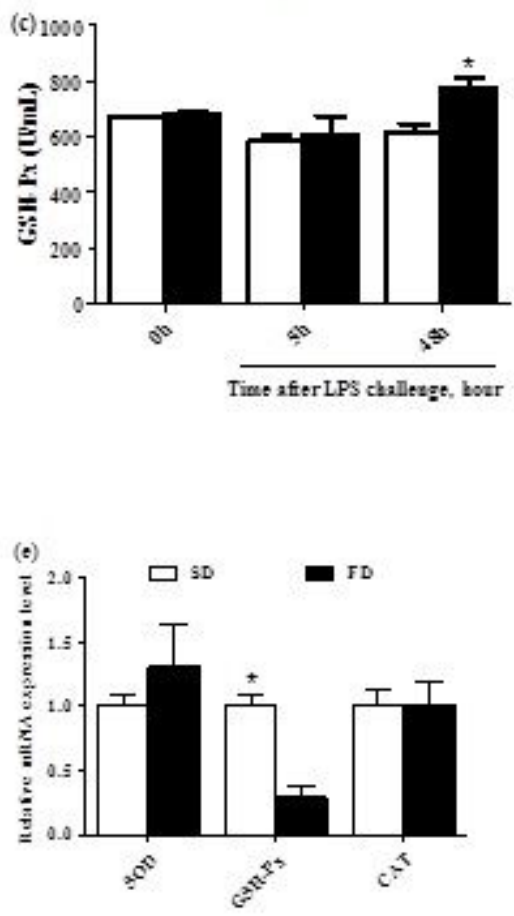

T-SOD

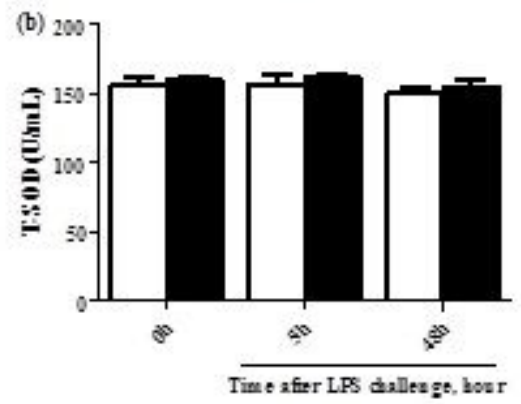

T-AOC

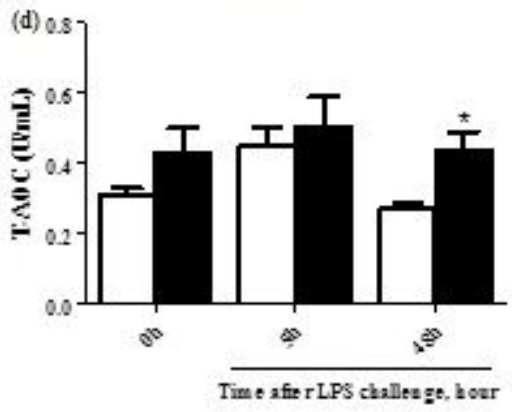

Figure 1

Effect of maternal diet with fish oil on the oxidative stress status in the piglets Effect of maternal diet with fish oil on the oxidative stress status in the plasma and livers of piglets. 8-iso-PG concentration (a), T-SOD activity (b), GSH-Px activity (c) and T-AOC (d) in plasma collected from piglets on 14d (14d), $14+5$ hour after LPS challenge (5h) ,and 14+48 hour after LPS challenge(48h) were determined. Relative expression levels of SOD, GPx and CAT mRNA in livers of piglets after 48h treatment of LPS collected from sows fed different diets (e) were determined by real-time PCR. Values from plasma are means $(n=6)$ and values from livers are means $(n=4)$, with their standard errors represented by vertical bars. *Mean values were significantly different between the two diet groups $(P<0.05)$. SD=Soybean oil diet, $F D=F i s h$ oil diet. 
INF-a

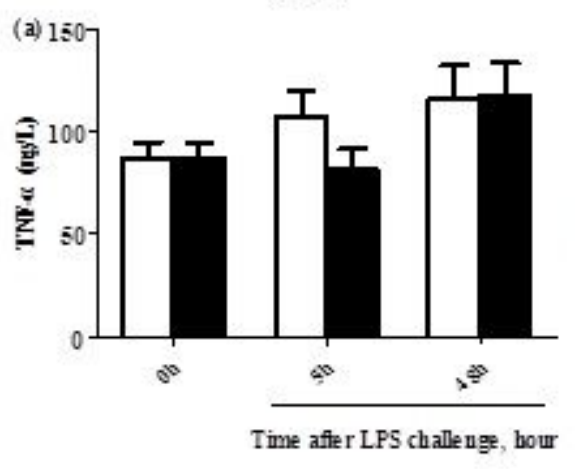

IL- 6
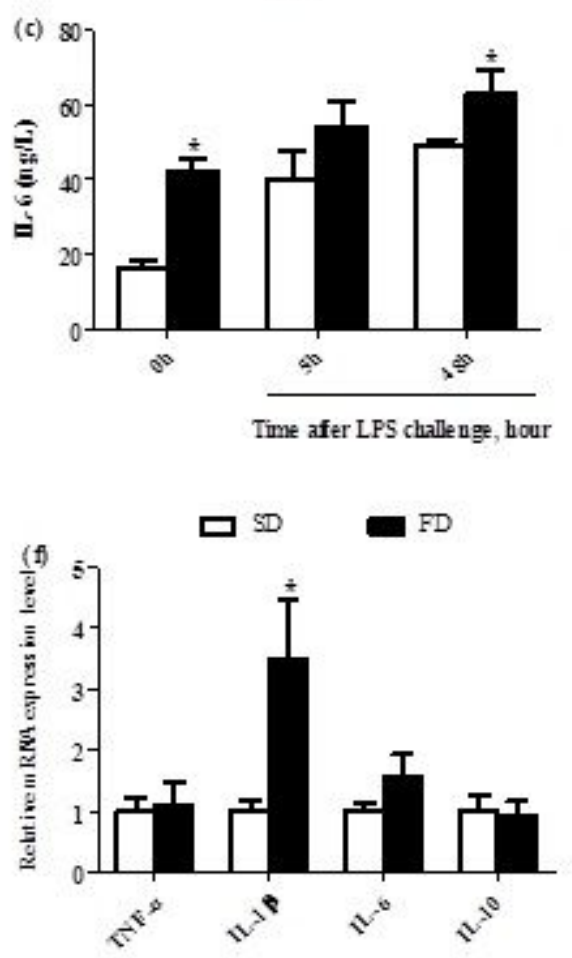

IL-1 $\beta$

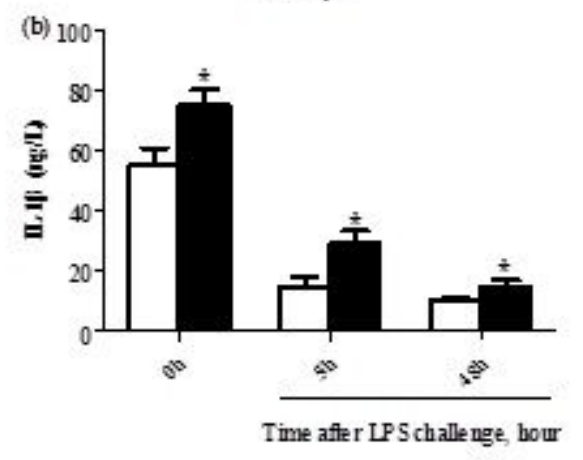

IL-10

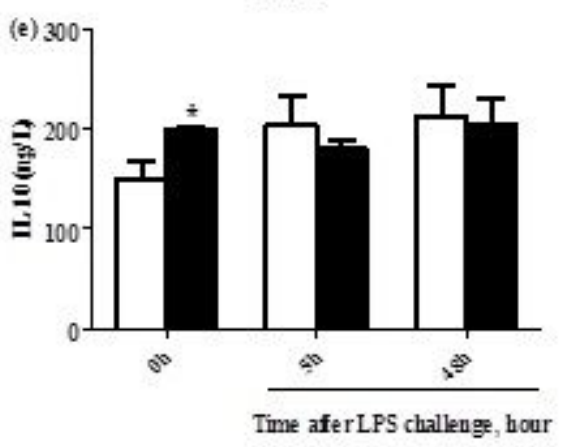

Figure 2

Effect of maternal diet with fish oil on the inflammatory response in the piglets Effect of maternal diet with fish oil on the concentrations of cytokines in plasma and mRNA expression of cytokines in the livers piglets. TNF-a concentration (a), IL-1 $\beta$ concentration (b), IL-6 concentration (c) and IL-10 concentration (d) in plasma collected from piglets on 14d (14d), 14+5hour after LPS challenge (5h), and 14+48 hour after LPS challenge(48h) were determined.Relative expression levels of IL-1 $\beta$, IL-6, and TNF-a and IL-10 mRNA in livers of piglets collected after 48h treatment of LPS collected from sows fed different diets (e) were determined by real-time PCR method. Values from plasma are means $(n=6)$ and values from livers are means $(n=4)$, with their standard errors represented by vertical bars. *Mean values were significantly different between the two diet groups $(P<0.05)$. SD=Soybean oil diet, FD=Fish oil diet. 

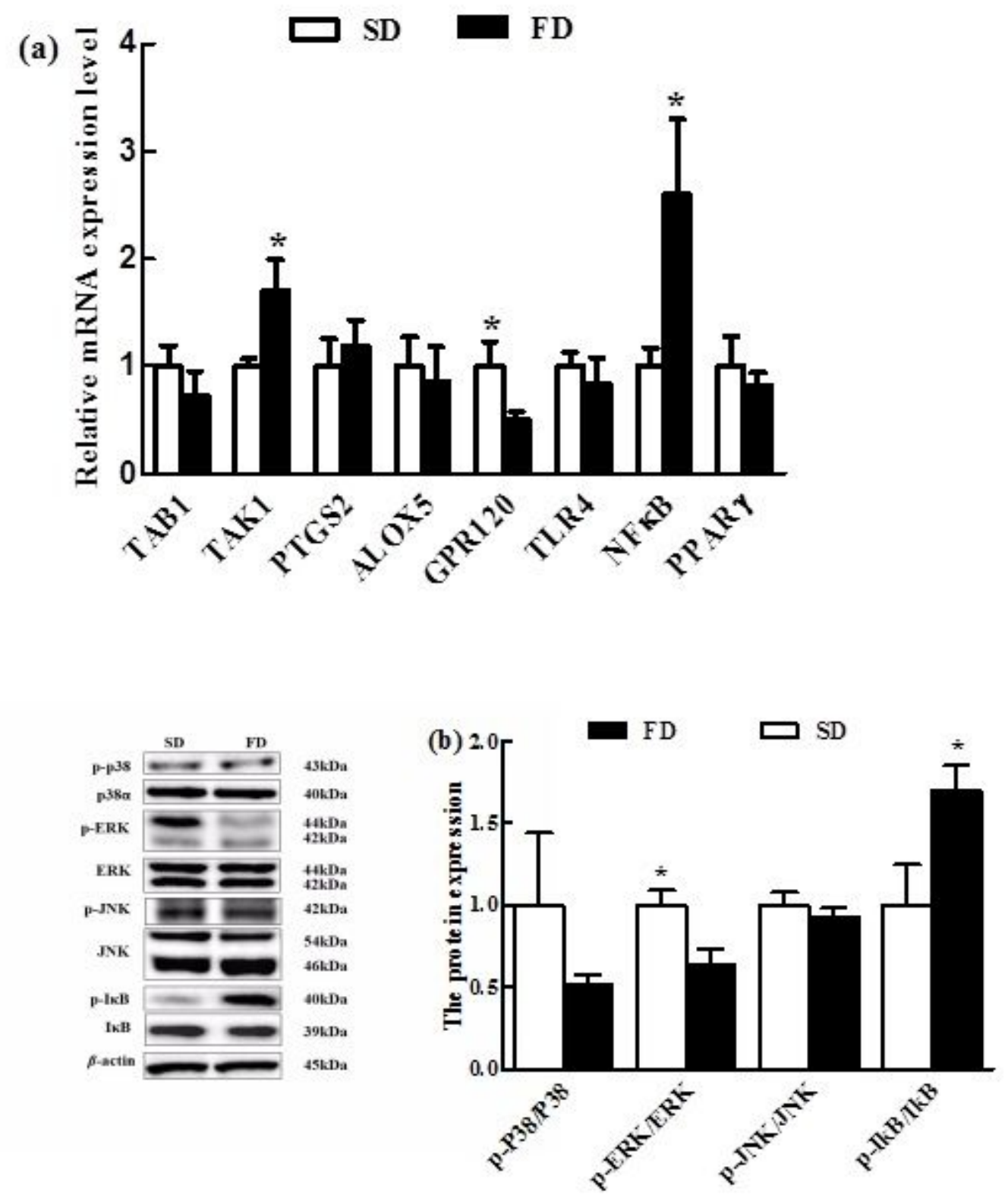

Figure 3

Effect of maternal diet with fish oil on mechanism parameters in the livers of piglets Effect of maternal diet with fish oil on mechanism parameters in the livers of piglets post-LPS challenged. (a) the expression of TAB1, TAK1, PTGS2, ALOX5, GPR120, TLR4, NF-KB and PPAR $y$ mRNA in the livers of piglets from sows fed different diets were detected by fluorescence quantitative PCR. (b)Western blotting method was used to detect the expression of JNK, p-JNK, p38 a, p-p38, ERK1/2, as well as phosphorylated p-ERK1/2, IKBa and $\mathrm{p}$-ІкBa protein in the livers of piglets from sows fed different diets. These values were expressed as the ratio of phosphorylated p-JNK, p-p38, p-ERK1/2 and P-IKB protein levels to JNK, p38, ERK1/2 and IKB protein levels. Values are means $(n=4)$, with their standard errors represented by vertical bars. *Mean values were significantly different between the two diet groups $(P<0.05)$. SD=Soybean oil diet, FD=Fish oil diet. 


\section{Supplementary Files}

This is a list of supplementary files associated with this preprint. Click to download.

- supplementarytable.docx

- supplementarytable.docx 